\title{
Heating and Evaporation of Droplets on a Super-hydrophobic Surface: Preliminary Results
}

\author{
Dmitrii Antonov', Roman Fedorenko', Pavel Strizhak ${ }^{1}$, Sergei S. Sazhin ${ }^{2}$ \\ ${ }^{1}$ National Research Tomsk Polytechnic University, 30 Lenin Avenue, Tomsk, 634050, Russia \\ antonovdv132@gmail.com; vfedrm@gmail.com; pavelspa@tpu.ru \\ ${ }^{2}$ Advanced Engineering Centre, School of Architecture, Technology and Engineering, University of Brighton, Brighton, \\ BN2 4GJ, UK \\ $\underline{\text { S.Sazhin@ brighton.ac.uk }}$
}

\section{Extended Abstract}

The importance of investigating the heating and evaporation of droplets on hydrophobic (contact angle $\sim 110^{\circ}$ ) and superhydrophobic (contact angle $\sim 160^{\circ}$ ) surfaces is well known [1]. Several models of the phenomena have been suggested and described in the literature [2,3]. All the models described so far, however, have been based on numerical solutions to the relevant transport equations and their applicability to the analysis of practical engineering problems is limited in many cases [3]. This abstract is focused on the preliminary results of the development of a new simple model of the phenomena. This model can capture the most important features of the processes and is simple enough for most engineering applications. The new model assumes that the contact area of the droplet with the superhydrophobic surface is much smaller than the area of the droplet surface. This assumption allows us to consider the droplet heating and evaporation as spherically symmetric processes. Heat supply to the droplet through the contact area of the droplet with the surface is considered as perturbation.

Heating of the droplet by ambient gas is described by a spherically symmetric heat conduction equation with the Robin boundary condition at the droplet surface. The effective thermal conductivity model, in which the effect of ambient air velocity is accounted for by the modification of the liquid thermal conductivity, was used. The analytical solution to the heat conduction equation is incorporated into the numerical code and used at each time step of the calculations [4]. The evaporation process is considered using the classical Abramzon and Sirignano model [4]. Heat supplied to the droplet through the contact area between the droplet and surface is calculated using the model described in [5]. The application of this model assumes that heat supplied to the droplet from the surface is instantaneously and homogeneously distributed throughout the whole droplet volume. Assuming that the surface temperature is not too high, the radiative heating rate of droplets is approximated as half of the radiative heating rate supplied by an external source (at the same temperature as the surface temperature) of radiation, surrounding the droplet. It is considered that the absorption of thermal radiation takes place inside the droplet volume (not at its surface [4]). Model predictions are shown to be consistent with in-house experimental data.

Work on this abstract was supported by the Russian Science Foundation (Grant 21-71-10008 and Grant 18-71-10002) (P. Strizhak, D. Antonov and R. Fedorenko; planning of the experiments, performing the experiment and applications of the models) and the Russian Science Foundation (Grant 21-19-00876) (S.S. Sazhin; development of the models).

\section{References}

[1] D. Hu, H. Wu, Z. Liu, "Effect of liquid-vapor interface area on the evaporation rate of small - sessile droplets', Int. J Thermal Science, vol. 84, pp. 300-308, 2014.

[2] S. Dash and S.V. Garimella, "Droplet evaporation on heated hydrophobic and superhydrophobic surfaces", Physical Review E, vol. 89, 042402, 2014.

[3] Z. Pan, S. Dash, J.A. Weibel, S.V. Garimella, “Assessment of wWtater $\underline{\text { d}}$ Droplet eEvaporation mAechanisms on h-hydrophobic and s-Superhydrophobic sSubstrates", Langmuir, vol. 29, pp. 15831-15841, 2013.

[4] S.S. Sazhin, Droplets and Sprays. Springer, 2014.

[5] P. Strizhak, R.S. Volkov, G. Castanet, F., Lemoine, F., O. Rybdylova, S.S. Sazhin, “'Heating and evaporation of - suspended water droplets: experimental studies and modelling’’, Int. J Heat Mass Transfer, vol. 127, pp. 92-106, 2018. 